\title{
Fortistis: Split Extension in Dense Passive Optical Networks by Inline Amplification with Remote ASE-Shaped Pump Delivery via Colorless Optical Network Units
}

\author{
Bernhard Schrenk*, Francesc Bonada, Jose A. Lazaro, and Josep Prat \\ Department of Signal Theory and Communications, Universitat Politecnica de Catalunya, \\ Jordi Girona 1, 08034 Barcelona, Spain (Tel. +34-93-401-7179 / Fax 7200) \\ *Corresponding author: bernhard.schrenk@tsc.upc.edu
}

\begin{abstract}
A method for extending the customer density via ASE-pumped splitters in the tree in a WDM/TDM-PON is presented. The influence of Rayleigh backscattering in the feeder is faced, originally prohibiting any upstream transmission.

(C)2010 Optical Society of America

OCIS codes: (060.2330) Fiber optics communications; (060.4250) Networks; (060.4230) Multiplexing
\end{abstract}

\section{Introduction}

Access networks have recently shown a migration towards the metro segment next to an increase in the data rate that is delivered to the customer. Cost effective passive optical networks (PON) are thereby extended in reach and split by wavelength division multiplexing (WDM) and time division multiplexing (TDM) [1], keeping their scalability and robustness [2]. As the power budget advances, remote amplification is taken into account to overcome optical losses [3]. Nevertheless, the induced optical signal-to-noise (OSNR) degradation limits the PON from being further expanded, if bidirectional data transmission via a single wavelength per customer, holding a wavelength-agnostic optical network unit (ONU) is targeted [4]. In addition, Rayleigh backscattering (RB) degrades the upstream performance for higher splitting ratios if there is a feeder fiber before the power splitter.

In this work, we aim for a technique of Fiber Optical Reamplification, Targeting Increased Split in the Tree by Injected noise Seed from the ONU. This approach, referred to as Fortistis, is shown to advance the power budget, where $8.5 \mathrm{~dB}$ can be gained, while the downstream launch can be reduced by $10 \mathrm{~dB}$ and the influence of $\mathrm{RB}$ is balanced between down- and upstream in the drop segment of a ring+tree PON. Besides, patterning in the semiconductor optical amplifier (SOA) based ONU is reduced.

\section{Partially Transparent Splitting in the Tree}

The high loss introduced by the high split in the tree, together with the generally higher noise figure of the ONU, leads to the strongest OSNR degradation for the upstream. In addition, the feeder fiber of the tree causes strong RB for the weak upstream due to the strong downstream. A more distributed amplification scheme with an Erbiumdoped fiber (EDF) placed inside the splitting stage, keeps the OSNR high. At the same time, the downstream can be launched with less power, leading to balanced optical signal-to-RB ratios (OSRR) for down- and upstream.

The pump for the EDF can be in principle provided by laser diodes in the ONU [5], though this introduces sources at the customer premises. Instead, we propose to reuse a SOA in a reflective ONU, initially intended to be a preamplifier/booster for down- and upstream [6], also as a pump for the EDF (Fig. 1). As a prerequisite, its gain spectrum has to reach the $1480 \mathrm{~nm}$ pump waveband. For the SOA used, the gain spectrum was centered at $1492 \mathrm{~nm}$, while a small signal gain of $23.3 \mathrm{~dB}$ and a noise figure of $6.4 \mathrm{~dB}$ are given at $1550 \mathrm{~nm}$.

A seed loop across the SOA, added via WDM couplers, provides the pump for the EDF. The amplified spontaneous emission (ASE) in the pump waveband is shaped into a specific range with a WDM multiplexer $\left(\mathrm{M}_{1}\right.$,

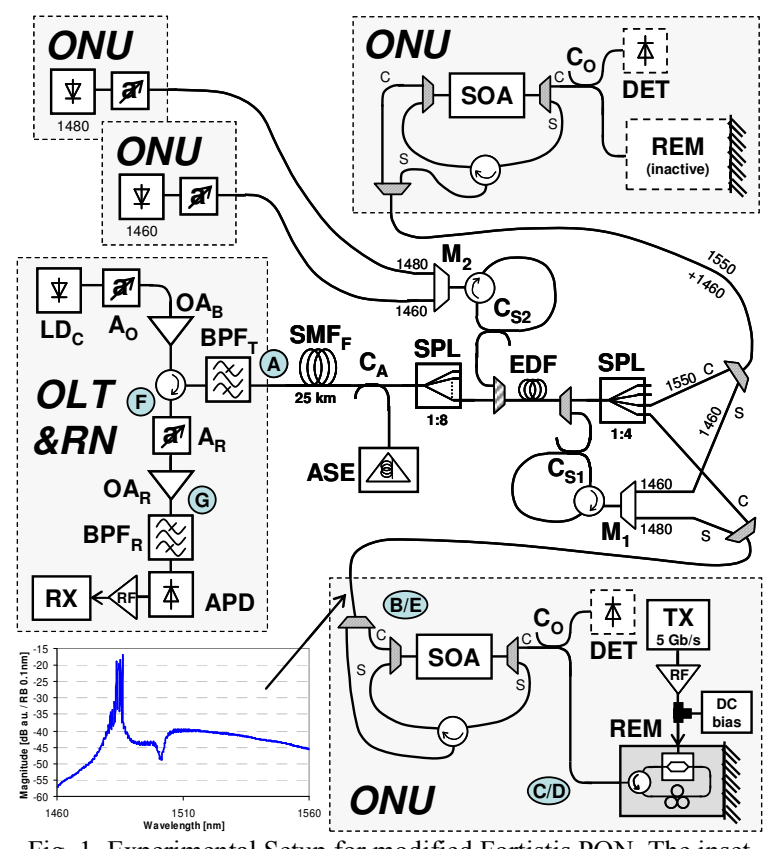

Fig. 1. Experimental Setup for modified Fortistis PON. The inset shows the spectrum of the ONU output without present data signal. 

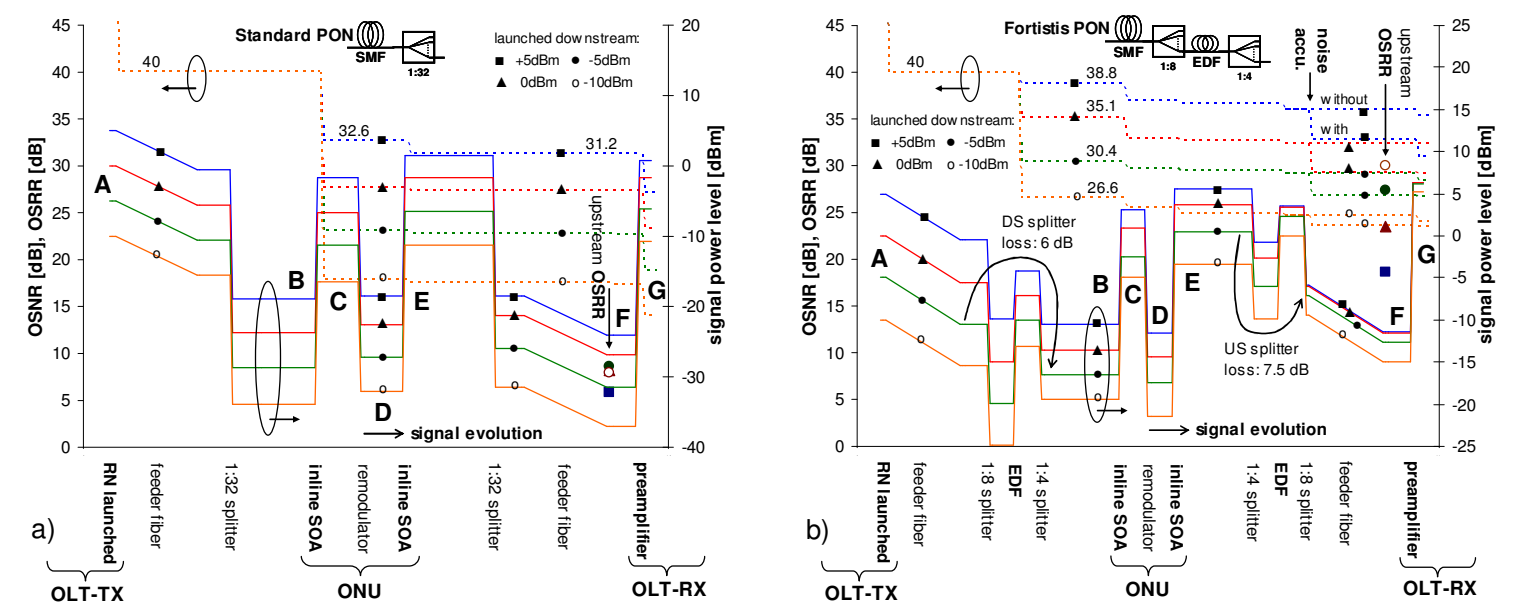

Fig. 2. Signal power (solid) and OSNR (dashed) for a) the standard PON and b) the modified Fortistis PON for the case with and without noise accumulation across the tree. The latter shows reduced dynamics due to the distributed amplification scheme, and raises the OSNR and OSRR.

$\mathrm{M}_{2}$ in Fig. 1) outside the ONU, to keep the latter colorless. This allows to combine the pumps of a few ONUs for a single EDF, in this case two 1460/1480 combiners allow four pumps for the EDF. With the losses in the loop and the feed-back to the SOA, provided by $20 / 80$ couplers $\left(\mathrm{C}_{\mathrm{S} 1}, \mathrm{C}_{\mathrm{S} 2}\right)$, a pump of $5.5 \mathrm{dBm}$ per ONU is obtained at the EDF input, shaped at 1480 and $1460 \mathrm{~nm}$. A $15 \mathrm{~m}$ long HE980 EDF with good pump conversion efficiency was inserted as the best choice between a 1:8 and 1:4 splitter stage. Two ONUs were seeding at 1480 and 1460 nm, while two laser diodes, with their output power adjusted to the obtained seed power, were emulating the two other ONUs at the 1:4 splitting stage.

As the SOAs in the ONUs which are not transmitting burst-mode upstream are active, noise accumulates across the tree. A coupler $\left(\mathrm{C}_{\mathrm{A}}\right)$ was therefore added with an ASE source, adjusted to the corresponding ASE of all other ONUs at the tree. Due to the ASE shaping with the seed loop, the noise density in the C-band is lower with the loop than for the solely SOA, ensuring that the ASE does not flood the tree.

\section{Results: Reduced Downstream Launch and RB Balancing}

The tree segment was fed from the remote node $(\mathrm{RN})$ with the downstream, having a variable launch power $\mathrm{P}_{\mathrm{RN}}$ but a fixed OSNR of $40 \mathrm{~dB}$, which is a typical value for this position inside the PON [6]. For comparison, a standard $P O N$ with the same feeder of $25 \mathrm{~km}$ standard single-mode fiber (SMF) and a 1:32 splitter was also evaluated. At the $\mathrm{ONU}$, a remodulator consisting of a Mach-Zehnder modulator was used after a 50/50 coupler $\left(\mathrm{C}_{\mathrm{O}}\right)$, with an extinction ratio better than $13 \mathrm{~dB}$. For evaluation, only the upstream was measured as it is passes the tree twice and is strongly degraded. No downstream modulation was present on the optical carrier at $1552.52 \mathrm{~nm}$, not to introduce additional errors deriving from an unsuppressed pattern. The upstream was modulated at 5 Gbps with a pseudorandom bit sequence (PRBS) of length $2^{31}-1$. This data rate was chosen to avoid additional errors due to handling of higher rates, as low penalties are subject of investigation. The receiver of the optical line terminal (OLT) comprised of an EDF amplifier pumped at $980 \mathrm{~nm}$ and an avalanche photodiode (APD). Several optical bandpass filters for ASE rejection had a bandwidth of $100 \mathrm{GHz}$.
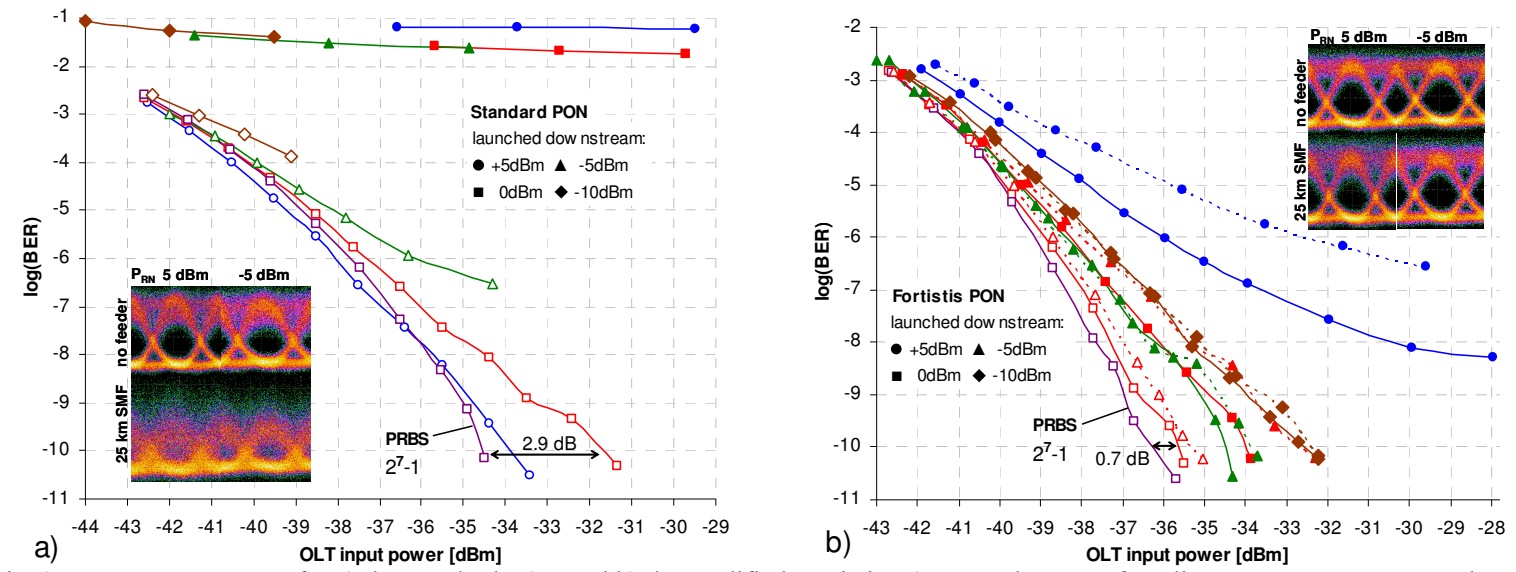

Fig. 3. BER measurements for a) the standard PON and b) the modified Fortistis PON. For the ease of reading, some curves are not shown. Measurements are taken for the case without (solid) and with (dashed lines) ASE accumulation, in the back-to-back (hollow) case and with feeder fiber (filled markers). Several curves correspond to a PRBS of length $2^{31}-1$ if not otherwise mentioned. 
The standard PON suffers from strong dynamics (Fig. 2a) due to the high splitter loss. A launched power of $0 \mathrm{dBm}$ is necessary to keep the OSNR higher than $25 \mathrm{~dB}$ after the first SOA pass. However, the OSRR is below $10 \mathrm{~dB}$ with present feeder fiber, making any transmission impossible (Fig. 3a). Even for the back-toback case, with fiber replaced by attenuation, the budget is too high to maintain transmission for a decreased launch without additional amplification in the tree.

The modified Fortistis PON has a smoothed signal evolution (Fig. 2b) due to the distributed amplification scheme. The splitter loss is reduced from the theoretical value of $15 \mathrm{~dB}$ to 6 and $7.5 \mathrm{~dB}$ for down- and upstream when a power of $-5 \mathrm{dBm}$ is launched from the RN. The OSNR is then $30.4 \mathrm{~dB}$ at the downstream reception, while it is 29.3 and $26.8 \mathrm{~dB}$ for the upstream without and with ASE accumulation at the tree. The OSRR can be maintained as high as $18.6 \mathrm{~dB}$ for a launched power of 5 $\mathrm{dBm}$, and it is bigger than $23 \mathrm{~dB}$ for $0 \mathrm{dBm}$ or less. A trade-off exists for the launched power regarding the OSNR and OSRR: the OSNR can be kept high with a strong downstream, however the OSRR decreases due to saturation effects in the amplifiers along the upstream path. For a lower launch, the OSRR is improved at the cost of the OSNR. In the modified PON, transmission is also possible with the feeder fiber (Fig. 3b). The penalty in sensitivity for a bit error ratio (BER) of $10^{-10}$,

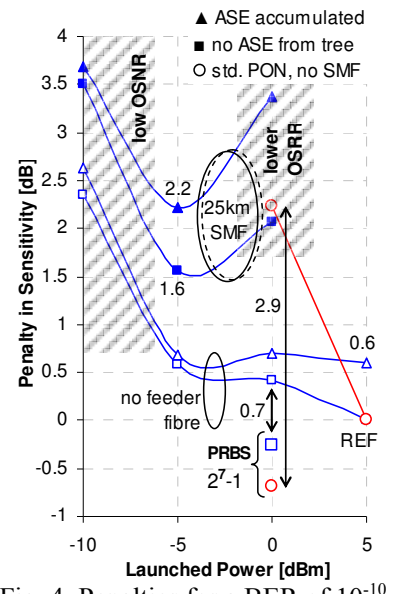

Fig. 4. Penalties for a BER of $10^{-10}$ referenced to the optimum launch in the back-to-back case. referenced to the back-to-back case with optimum launch, is shown in Fig. 4. The optimum levels were $5 \mathrm{dBm}$ for the back-to-back cases, due to the high OSNR and the absence of RB, and $-5 \mathrm{dBm}$ for the Fortistis PON with feeder fiber, having a penalty of $1.6 \mathrm{~dB}$ and additional $0.6 \mathrm{~dB}$ caused by ASE accumulation across the tree. A BER floor is only present for the highest launch of $5 \mathrm{dBm}$. From the values obtained, the downstream OSRR has been found to be $>20 \mathrm{~dB}$ for a launched power of $-5 \mathrm{dBm}$ and is therefore the lower one compared to the upstream OSRR after balancing.

Thanks to the seed loop, the carrier density in the SOA is clamped. The patterning penalty between a PRBS of length $2^{7}-1$ and $2^{31}-1$, deriving from the transient distortions introduced by the slow gain saturation of the SOA, is reduced to $0.7 \mathrm{~dB}$ for a launch of $0 \mathrm{dBm}$, compared to $2.9 \mathrm{~dB}$ of the standard PON.

\section{Discussion: Extension Capabilities for a Hybrid WDM/TDM-PON}

With the assumed conditions between OLT and RN, having an OSNR of $40 \mathrm{~dB}$ and an optimum launch of $-5 \mathrm{dBm}$, power margins of 8 and $21.2 \mathrm{~dB}$ for down- and upstream can be reached. These margins can be further increased by remote amplification in the $\mathrm{RN}$, providing margins above $23 \mathrm{~dB}$ when a gain of $15 \mathrm{~dB}$ is assumed in the $\mathrm{RN}$ [3].

This would correspond to a ring reach of $50 \mathrm{~km}$ and an additional split of 1:16, forming with 32 wavelengths a PON that can serve $16.3 \mathrm{k}$ customers. Although this high split of $32 \times 32$ means a lower guaranteed data rate per user, it is promising for very short reach PONs with in future increased data rates.

\section{Conclusion}

A method for reducing the loss in the tree splitter was demonstrated by remote pumping of an intermediate amplification stage in the tree from the ONU, facing also the problem of RB caused by the feeder fiber. No additional light sources have been added to the colorless customer premises equipment. For a tree with $25 \mathrm{~km}$ and a splitting of 1:32, the net splitter loss was reduced from $16 \mathrm{~dB}$ to around $7.5 \mathrm{~dB}$, allowing at the same time a reduced downstream launch of $-5 \mathrm{dBm}$ from the remote node into the tree segment. This in turn raises the optical signal-to$\mathrm{RB}$ ratio to $>27 \mathrm{~dB}$, while it would be $<10 \mathrm{~dB}$ for the standard configuration. Besides, the pump power requirements for the case of remote amplification in the PON can be relaxed due to a weaker downstream launched from the RN.

Acknowledgement: This work was supported by the European FP7 SARDANA project and the Spanish MICINN project TEC2008-01887.

\section{References}

[1] L.G. Kazovsky, W. Shaw, D. Gutierrez, N. Cheng, S. Wong, "Next-Generation Optical Access Networks,” J. of Lightwave Technology 25, 3428-3442 (2007).

[2] J.A. Lazaro, C. Bock, V. Polo, R.I. Martinez, J. Prat, "Remotely amplified combined ring-tree dense access network architecture using reflective RSOA-based ONU," J. of Optical Networking 6, 801-807 (2007).

[3] J.M. Oh, S.G. Koo, D. Lee, S.J. Park, "Enhancement of the Performance of a Reflective SOA-Based Hybrid WDM/TDM PON System With a Remotely Pumped Erbium-Doped Fiber Amplifier," J. of Lightwave Technology 26, 144-149 (2008).

[4] B. Schrenk, F. Bonada, M. Omella, J.A. Lazaro, J. Prat, "Enhanced Transmission in Long Reach WDM/TDM Passive Optical Networks by Means of Multiple Downstream Cancellation Techniques," Proc. ECOC'09, We.8.5.4 (2009).

[5] M.D. Feuer, et al, "Remotely-pumped self-amplified star network for local access," Proc. OFC'96, 146-147 (1996).

[6] B. Schrenk, et al, "C+L Band Remote Node for Amplification in Extended Reach Full-Duplex 10Gb/s WDM/TDM Passive Optical Networks," Proc. ECOC’09, We.P6.19 (2009). 